\title{
SOME CONSEQUENCES OF THE APPROXIMATION THEOREM OF BING
}

\author{
O. G. HARROLD, JR. ${ }^{1}$
}

1. Introduction. In the following paragraphs we consider several unsolved problems in the topology of 3-space. Among the results is included a new formulation of properties characterizing tame curves in three-space without any explicit mention of polyhedral sets.

Three-space is denoted by $R^{3}$, the null-set by $\square$, and the set of points in $A$ but not in $B$ by $A \backslash B$. The combinatorial boundary of chain $E$ is $\partial E$ and the set of points on such a boundary by $|\partial E|$. The closure of a set $A$ is denoted by $\mathrm{Cl} A$, or $\bar{A}$.

Theorem (Bing). Given a compact 2-manifold $M$ of $R^{3}$ with or without boundaries, a compact subset $D$ of $R^{3}$ and a positive number $\epsilon$. There is a 2-manifold $N$ and a homeomorphism $f$ of $M$ on $N$ such that no point of $M$ is moved more than $\epsilon$ and $N$ is locally polyhedral at each point of $N \backslash D$. If $D \neq \square, f$ may also be taken so that no point $x$ is moved more than the minimum of $\epsilon$ and the distance from $x$ to $D$. In particular, $f$ is the identity map at all points of $D \cap M$ and $D \cap M=D \cap N[1]$.

2. The "orthogonal" disk problem. Some simple closed curves in $R^{3}$ have the property that, if $J$ is the curve, there is a disk $D$ whose boundary links $J$ and such that $J$ and $D$ meet in a single point. This property is preserved by homeomorphisms acting on $R^{3}$. The class of curves having this property is known to include curves $\{J\}$ such that under some homeomorphism of $R^{3}$ on itself some sub-arc of $J$ is mapped on a rectifiable arc [5]. By the use of his approximation theorem, R. H. Bing [2] has constructed an example of a curve that admits no orthogonal disk.

3. A new formulation of properties $\odot$ and $Q$. A 1 -manifold $J$ is said to have property $\odot$ if at each point $x$ of $J$ and for positive $\epsilon$ there is a set $K(x, \boldsymbol{\epsilon})$ of diameter less than $\epsilon$ that is a topological 2 -sphere whose interior contains $x$, that meets $J$ at a set of points whose cardinal does not exceed the order of $x$ in $J$, and such that $K$ is locally polyhedral modulo $J$.

To see that the locally polyhedral character of $K$ may be omitted

Presented to the Society, November 15, 1955; received by the editors March 10, 1956.

1 This paper was written at the Point Set Topology Institute (University of Wisconsin, 1955) which was supported by a National Science Foundation Grant. 
suppose $K$ has the other requirements and $\epsilon^{\prime}<\epsilon-$ diameter $K$. Using $M=K, D=J$ and $\epsilon^{\prime}$ in the approximation theorem, then $N=K_{1}(x, \epsilon)$ has the original requirements.

A 1 -manifold $J$ is said to have property $Q$ at $x$ if there is a disk $D_{1}$ such that $J \cap D_{1}$ is the closure of a neighborhood of $x$ in $J$ and $D_{1}$ is locally polyhedral at the points of $D_{1} \backslash J$. To see that the locally polyhedral character of $D_{1}$ may be omitted, suppose $D_{2}$ has the requirements of $D_{1}$ apart from locally polyhedral character. If $M=D_{2}$ and $D=J$ and $\epsilon$ arbitrary in the approximation theorem we find a disk $N=D_{1}$ that is locally polyhedral at points of $N \backslash J$ and has the other requirements.

4. 1-cells contained in the interior of 2-cells. It has been known since 1921 when Antoine's thesis appeared that an arc in $R^{3}$ may be a subset of no topological 2-cell in $R^{3}$. Such an arc certainly does not lie on the boundary of a 2-cell and still less on the boundary of a 3cell. (That a 2 -cell in $R^{3}$ may be a subset of no topological 2 -sphere and hence lie on the boundary of no 3-cell is implied by a result announced by Kapuano [7]). We limit ourselves here chiefly to the special case where the 1-cell lies interior (relative) to a 2-cell.

A fairly simple proof is now available that arcs exist lying on no 2cell. Example 1.4 of Fox-Artin [3] is an example of a wild arc that is the union of two tame arcs, having only an end point in common. If $J$ is the set of points on this arc and $J_{1}$ is a sub-arc of $J$ whose interior contains the "wild point" of the imbedded arc, then $J_{1}$ is also wild. Now if $J$ were a part of the boundary of some disk $D$ in $R^{3}$, then $J_{1}$ has properties $\odot$ and $\mathcal{Q}$ (in the new form) and, by Theorem 6 of [6], would be tame. But $J_{1}$ is wild.

THEOREM. Let $J$ be a closed 1-cell in $R^{3}$, then $J$ lies in the interior of a closed 2-cell if and only if $J$ lies on the boundary of some bounded, open, connected ulc ${ }^{1}$ subset of $R^{3}$.

If: Let $A$ be the bounded, open, connected subset of $R^{3}$ that is $u l c^{1}$ and has $J$ on its boundary. By the results of Wilder, [8, Theorem 8.3, p. 311], every nondegenerate component of the boundary $M$ of $A$ is a classical 2-manifold. If $M_{1}$ is the component of $M$ determined by $J$, then, by triangulation of $M_{1}$, an open 2-cell $E$ in $M_{1}$ may be found such that $J$ is in Int $E$ (rel $M_{1}$ ).

Only if: If $J$ lies in the interior of a disk $E$, there is a sub-disk $E_{1}$ of $E$ such that $J$ is in $\left|\partial E_{1}\right|$. Using the approximation theorem (with $D=J, M=E_{1}$ ) there is an "almost" polyhedral disk $N$ whose boundary contains $J$ that is locally polyhedral at points of $N \backslash J$. One may "inflate" $N$ to obtain a closed 3-cell $F$ that is locally polyhedral at 
points $F \backslash J$ and $|\partial F|$ contains $J$. Then $J$ is contained in the closure of the interior of $F$ and Int $F$ is the required bounded, open, connected, ulc ${ }^{1}$ subset.

5. A special case of the union of two locally tame sets. It is known that locally tame sets are tame. However, the union of two tame sets having a single common point need not be tame. In this connection the following theorem is of interest.

THEOREM. Let $J$ be a simple closed curve that is polyhedral and let $J$ be the boundary of any disk $F$ in $R^{3}$. Then $J$ is the boundary of a polyhedral disk.

(Thus $J$ is tame and unknotted).

Proof. If $J$ is the boundary of any disk $D$, then $J$ has the (new) property $Q$ at each point. Since $J$ is polyhedral, it has property $P$ at each point. By Theorem VII of [6] it is locally tamely imbedded, hence tamely imbedded. By Graeub [4, p. 39], this implies there is a polyhedral 2-cell of which $J$ is the boundary.

\section{REFERENCES}

1. R. H. Bing, Approximating surfaces with polyhedral ones, Bull. Amer. Math. Soc. Abstract 61-6-824. (A sketch of the proof may be found in the Proceedings of the Point Set Topology Institute, University of Wisconsin, 1955).

2. - A simple closed curve which pierces no disk, Bull. Amer. Math. Soc. Abstract 62-1-156.

3. R. Fox and E. Artin, Some wild cells and spheres in 3-dimensional space, Ann. of Math. vol. 49 (1948) pp. 979-990.

4. W. Graeub, Die semi-linearen Abbildungen, Sitzungsberichte der Heidelberger Akad., 1950, Part 4.

5. O. G. Harrold, Jr., A theorem on disks, Proc. Amer. Math. Soc. vol. 7 (1956) pp. 153-154.

6. O. G. Harrold, Jr., H. C. Griffith and E. E. Posey, A characterization of tame curves in 3-space, Trans. Amer. Math. Soc. vol. 79 (1955) pp. 12-35.

7. I. Kapuano, Sur les surfaces homeomorphes a un disque dans un $R^{8}, \mathrm{C} . \mathrm{R}$. Acad. Sci. Paris vol. 256 (1953) pp. 1229-1230.

8. R. L. Wilder, Topology of manifolds, Amer. Math. Soc. Colloquium Publications, vol. 32, 1949.

The University of Tennessee 